\title{
Design of One-Dimensional Composite Photonic Crystals with an Extended Photonic Band Gap
}

\author{
Vladimir Tolmachev \\ Trinity College Dublin \\ Kevin Berwick \\ Technological University Dublin, kevin.berwick@tudublin.ie
}

Follow this and additional works at: https://arrow.tudublin.ie/engscheceart

Part of the Electrical and Electronics Commons

\section{Recommended Citation}

Tolmachev, V.A., Perova, T.S. and Kevin Berwick: Design of One-Dimensional Composite Photonic Crystals with an Extended Photonic Band Gap. Journal of Applied Physics, Vol. 99, issue 3, 2006. doi:10.1063/ 1.2165401

This Article is brought to you for free and open access by the School of Electrical and Electronic Engineering at ARROW@TU Dublin. It has been accepted for inclusion in Articles by an authorized administrator of ARROW@TU

Dublin. For more information, please contact

arrow.admin@tudublin.ie, aisling.coyne@tudublin.ie, gerard.connolly@tudublin.ie.

Funder: Science Foundation Ireland

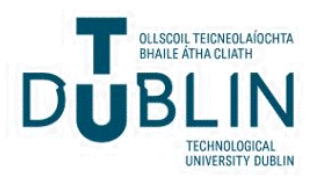




\title{
Design of one-dimensional composite photonic crystals with an extended photonic band gap
}

\author{
V. A. Tolmachev ${ }^{\text {a) }}$ and T. S. Perova ${ }^{\text {b) }}$ \\ Department of Electronic and Electrical Engineering, University of Dublin, Trinity College, \\ Dublin 2, Ireland \\ K. Berwick \\ Department of Electronic and Communications Engineering, Dublin Institute of Technology, Kevin Street, \\ Dublin 8, Ireland
}

(Received 22 August 2005; accepted 9 December 2005; published online 2 February 2006)

\begin{abstract}
A technique for photonic band gap (PBG) extension based on mixing photonic crystals with different lattice constants or filling factors is suggested. For the design of photonic crystals with maximal PBG extension the gap map imposition method is utilized. Gap maps for composite photonic crystals based on Si-air structures are calculated and used to predict optimal structures for fabrication. (C) 2006 American Institute of Physics. [DOI: 10.1063/1.2165401]
\end{abstract}

\section{INTRODUCTION}

Photonic crystals (PCs) are materials with a periodic modulation of the dielectric constant. This modulation gives rise to what is known as a photonic band gap (PBG) within the crystal. ${ }^{1}$ It is important to know the width of the PBG within the structure and, in general, it is desired to have a band gap as wide as possible during the design of a PC. One method of determining the band gaps within photonic crystals is to analyze the dispersion curves. ${ }^{2}$ This allows the gap maps of PBGs, ${ }^{2}$ a graphical representation of the PBG width as a function of the filling factor $f$ (the fraction of one of the components in the lattice constant of the crystal) to be obtained.

For one-dimensional (1D) PCs there is an alternative approach available to generate the gap map. Using the transfer matrix method, ${ }^{3,4}$ the set of reflection spectra for the $f$ values in the range from 0 to 1 is calculated. The wavelengths $\lambda$ (or wave numbers, $\widetilde{\nu}$ ) for which $R=1$ is determined and this information is then used to generate the gap map. ${ }^{5}$ The PBG gap map allows an effective comparison of PC structures with various periods $m$, lattice constant $A$, refractive index contrast $\Delta n=n_{1} / n_{2}$, angle of incidence and polarization of the incident light. ${ }^{2,5,6}$ The gap map is a valuable tool for use during the specification of a $\mathrm{PC}$ for a particular application.

In general, an extension or widening of the PBG can be achieved by increasing the optical contrast $\Delta n$ and the number of the lattice periods $m$. Recently a number of methods for the extension of the high reflection range (HRR) for dielectric multilayer stack structures consisting of binary layers has been suggested based on a combination of experimental observation and theoretical considerations. ${ }^{7-9}$ The theoretical framework for these methods was developed as a result of studies of light localization in 1D systems. ${ }^{10-12}$ These theoretical studies show that if disorder is introduced to a peri-

\footnotetext{
a) Also at Ioffe Physico-Technical Institute, Politechnicheskaya 26, St. Petersburg, Russia.

b) Author to whom correspondence should be addressed; electronic-mail: perovat@tcd.ie
}

odic multilayer system, light of any wavelength can be localized. Zhang et $a l^{7}$ have shown both theoretically and experimentally that for $\lambda / 4$ multilayers with high refractive indices and an optical contrast $\Delta n=2.83 / 1.33$, it is possible to extend the PBG. In order to cause this extension, disorder was introduced to the higher refractive index layers as a thickness variation along the whole structure. In Ref. 8 the authors performed PBG calculations for the structure with disordered binary layers and an optical contrast $\Delta n$ $=2.3 / 1.45$. The calculations were confirmed by experiment which shows a HRR extension between 1.5 and 1.85 times. This approach was further developed in Ref. 9, where it was shown theoretically that PBG extension in 1D PCs can be even more substantial, up to three times wider, if three neighboring PBGs can be merged into one PBG. In order to achieve this level of PBG enlargement, the authors suggest using not only $\lambda / 4$ layers together with a high optical contrast $(\Delta n=3.2 / 1.38)$, but also a Gaussian distribution of thickness disorder. In Ref. 13 the authors designed and fabricated a 1D PC based on Si-air components with an even larger optical contrast $(\Delta n=3.42 / 1)$. The total number of lattice periods was ten, however, disorder was introduced into only three Si layers. Even this relatively small amount of disorder resulted in the merging of three neighboring PBGs into one in the calculated spectrum. Experimental measurements of the reflection spectra ${ }^{13}$ in the midinfrared range confirmed these theoretical predictions.

Another suggestion for the extension of the spectral region of the HRR has been made in Ref. 14. The author suggested to use a periodic structure with the optical thickness equal to $\lambda / 4$ designed for the certain wavelength, which followed by another structure so as its HRR will join the previous one and thus increase the resulting reflection band of the composite PC. Therefore, the discrete, but not a disordered one, composition of periodic structures is suggested in Ref. 14

In this work we propose to use a similar idea but with different approach. This approach is based on using the overlapping areas of the gap map in order to determine the com- 

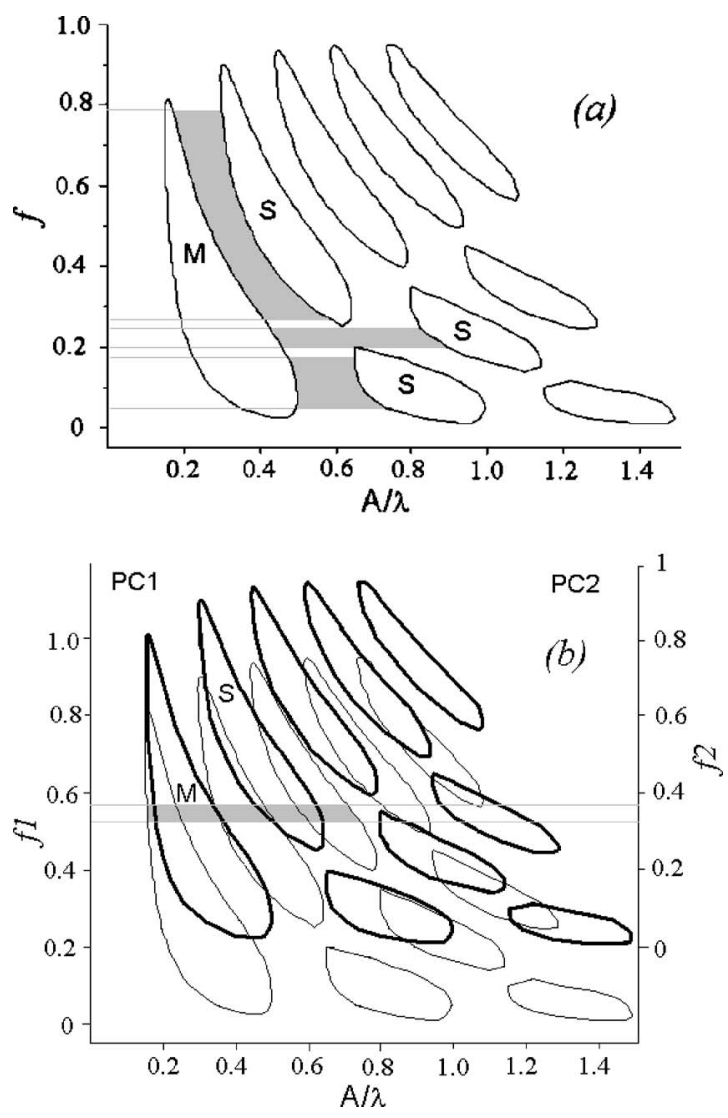

FIG. 1. (a) The gap map of PBGs and transparent regions (gray shaded area), where the light must be localized in order to form an extended PBG with inclusion of the main $(\mathrm{M})$ band gap, for a Si-air system with $n_{\mathrm{Si}}$ $=3.42, n_{\text {air }}=1, m=10$, and $R_{\mathrm{PBG}}=0.999$. (b) Overlapping of two PBG gap maps (from two conventional PCs, viz. PC1 and PC2) with a shift of the filling factor by $\Delta f=0.2$. Two PBG regions of PC2 (thick lines) for $f 2$ $=0.31-0.34$ covering two transparency regions of PC1 (thin lines) with $f 1$ $=0.51-0.54$. The range of $f$ values correspond to the maximum region of EPBG $\Delta(A / \lambda)=0.57$.

posite extended PBG. Our approach visually demonstrates the possibility of designing periodic structures of arbitrary optical thickness by varying both the lattice constant and the filling factor. Various structures and their gap maps for the composite PCs are presented, illustrating the usefulness of the technique.

\section{CALCULATION OF THE BAND GAP EXTENSION}

In order to illustrate the construction of a gap map, we will use, as an example, a periodic $\mathrm{Si}$-air structure with an optical contrast $\Delta n=3.42 / 1$, i.e., $n_{\mathrm{Si}}=3.42$ and $n_{\text {air }}=1$. The first step is to calculate the reflection spectra, $R$ for this structure using the transfer matrix method ${ }^{3,4}$ under normal incidence of light. The structure under consideration is taken to have ten periods, so $m=10$, and we will use $R>0.999$ (i.e., $R_{\mathrm{PBG}} \approx 1$ ) as the criterion for band gap formation. For the purpose of these calculations the light beam is assumed to enter the PC from an ambient with $n=1$ and, after traveling through the PC to reenter the same ambient. The calculated PBG regions are presented in Fig. 1(a) as closed regions, the empty space surrounding these regions represent the ranges of transmission, $T$. Note that transmission means the regions of wavelength where $T$ can vary from 0.001 up to 1 . The main $\mathrm{PBG}$, labeled $\mathrm{M}$, together with the closest secondary PBGs, labeled S, are shown in Fig. 1. Now we will estimate the distance between the PBG regions for different values of $f$. There are three ranges of $f$ of interest to us, since these ranges include both the main and secondary PBGs. The ranges of transmission corresponding to these $f$ values are represented by the gray shaded area in Fig. 1(a). The extension of the $T$ regions, in units of $A / \lambda$, is from 0.17 to 0.25 for $f=0.05-0.18$; from 0.4 to 0.43 for $f=0.2-0.25$; and from 0.11 up to 0.13 for $f=0.27-0.78$. For PBG extension the most appropriate region of $f$ is $f=0.27-0.78$ and the most inappropriate region corresponds to $f=0.2-0.25$. It is worth noting that $\lambda / 4$ layers, for which $f=0.226$ here, which are commonly used for the fabrication of 1D PCs with nonextended PBGs, are located in this unfavorable region of $f$. In other words, merging the PBGs for this particular region of $f$, where light needs to be localized in the region of $A / \lambda$ from 0.4 to 0.43 , would be the most difficult.

How we can localize the incident light in the transmission regions? As discussed earlier in Ref. 9 the authors were able to merge three PBGs by introducing thickness disorder into one of the alternating layers. It is known that the variation in the thickness of one of the layers is equal to the change in the lattice constant for this particular layer within the structure. In Ref. 8 the disorder was modeled by varying the thickness in both alternating layers, which is equivalent to the introduction of an additional parameter viz. the filling factor. So, we can determine the PBG extension by varying these parameters. For example, instead of introducing disorder as an extended thickness variation, that is, a variation in lattice constant or filling factor, we could introduce another PC with different parameters. In order to estimate the influence of the combination of all these parameters on the properties of the composite PC we will use gap map analysis.

\section{A. Comparison of photonic crystals with the same $A$ and different $f$}

Let us assume that we have two photonic crystals, PC1 and PC2 with the same lattice constant $A$ and number of periods $m$. We take the gap map obtained for PC1 with the PBGs presented in Fig. 1(b) by thin lines, and we move it along the $Y$ axis up with respect to the original gap map. By doing this we are moving the PBGs of PC2 so that the transmission regions for $\mathrm{PC} 1$ will also be covered by the PBGs of PC2. Since we are not shifting the gap map along the $X$ axis, the values of $A / \lambda$ for both PCs remain unchanged. Performing a relative shift of $\Delta f$ from 0 to 1 , we found the merging of the PBGs for both PCs with a maximum extension occurs when the values of $f$ are $f 1=0.51-0.54$ and $f 2=0.31-0.34$ for $\mathrm{PC} 1$ and $\mathrm{PC} 2$, respectively, with the value of $\Delta f=0.2$. Thus, two PBG regions for PC2 cover two transmission regions of $T$ from the original PC1 [ Fig. 1(b)]. Now we can state that the composite $\mathrm{PC}$ constructed from the two photonic crystals $\mathrm{PC} 1$ and $\mathrm{PC} 2$ will have an extended PBG (EPBG) in the region of $A / \lambda$ from 0.16 all the way up to 0.73. Therefore, if the maximum width of the PBG for the 

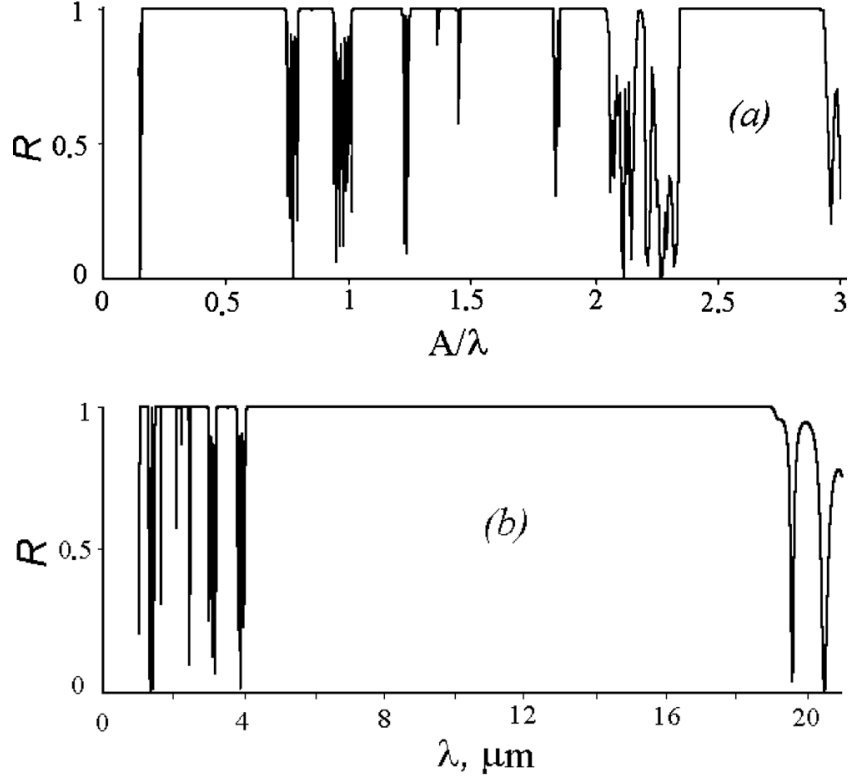

FIG. 2. Reflection spectra of composite PC with shift of the filling factor by $\Delta f=0.2(f 1=0.53, f 2=0.33) . n_{\mathrm{Si}}=3.42, n_{\text {air }}=1, m=10$, and $R_{\mathrm{PBG}}=0.999$. (a) Spectrum $R$ presented in relative units $(A / \lambda)$. EPBG width is $\Delta(A / \lambda)$ $=0.73-0.16=0.57$. Note the additional EPBG with almost the same width due to the merging of the secondary PBGs in the region $A / \lambda=2.3-2.9$. (b) Spectrum $R$ presented in wavelength units for $A=3 \mu \mathrm{m}$. The width of the EPBG is $\Delta \lambda=19.1-4.3=14.8 \mu \mathrm{m}$.

1D PC for $f=0.226$ was $\Delta(A / \lambda)=0.44-0.2=0.24$, the width of the EPBG is $\Delta(A / \lambda)=0.73-0.16=0.57$, i.e., a factor of 2 wider.

Now, let us design a composite photonic crystal (CPC) based on PC1 and PC 2 with the same lattice constant $A$. For this calculation, we choose a lattice constant $A=3 \mu \mathrm{m}$ and the number of periods $m=10$ for each PC. Therefore, the total number of periods for the CPC is $m=20$. We choose $f 1=D 1_{\mathrm{Si}} / A=0.53$ for PC1 while for PC2 $f 2=0.33$ bearing in mind that the light enters PC2 first. The thickness of the $\mathrm{Si}$ layer for PC1 and PC2 are $D 1_{\mathrm{Si}}=1.59$ and $D 2_{\mathrm{Si}}=0.99 \mu \mathrm{m}$, respectively. The reflection spectra for this $\mathrm{CPC}$ obtained using the transfer matrix method as described above is shown in Figs. 2(a) and 2(b). As can be seen from Fig. 2(a) the reflectance $R$ reaches values of greater than 0.999 in the regions of $A / \lambda=0.16-0.73$, confirming the formation of the EPBG. The PBG's width is $\Delta(A / \lambda)=0.57$ and is in the region of $\lambda=4.3-19.1 \mu \mathrm{m}$ from Fig. 2(b), which agrees with the predicted PBG width obtained from the gap map [Fig. 1(b)]. This method of determining the photonic properties of $\mathrm{CPC}$ by combining the properties of the individual photonic crystals is confirmed by our calculations of the reflection spectra of a CPC.

\section{B. Comparison of composite PC with different $\boldsymbol{A}$ and the same $f$}

A simple shift of the gap map along the $X$ axis cannot be used here, because $A$ is different for each PC in this case. In order to address this difficulty, the gap map of PBGs for this case is presented in wave number $(\widetilde{\nu}=1 / \lambda)$ units, rather than $A / \lambda$ units, for a particular value of $A$. We choose the lattice
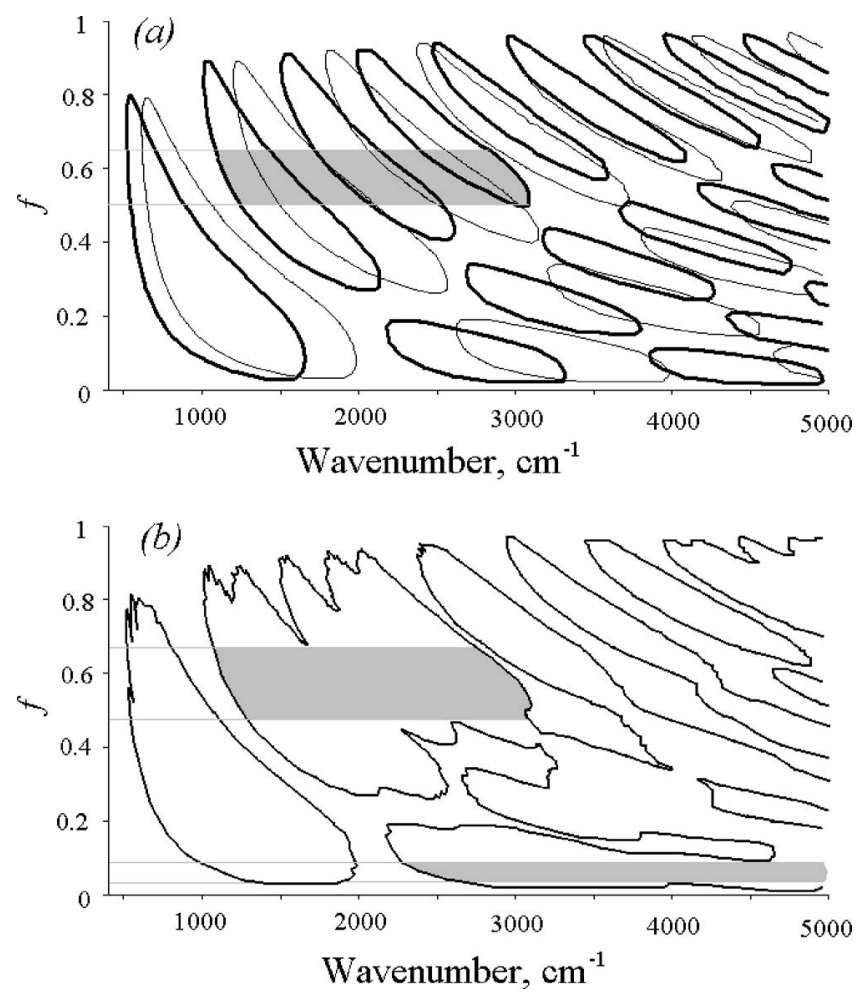

FIG. 3. (a) Overlapping gap maps for two PCs with lattice constants $A 1$ $=3 \mu \mathrm{m}$ (thick lines) and $A 2=(0.83) A 1=2.5 \mu \mathrm{m}$ (thin lines). $n_{\mathrm{Si}}=3.42, n_{\text {air }}$ $=1, m=10$, and $R_{\mathrm{PBG}}=0.999$. In the region of $f=0.6$ the EPBG region consisting of five overlapped PBGs from a conventional PC is shown. (b) PBG gap map for composite PC, the extended PBGs are in the range of $f=0.06$ and $f=0.6$.

constant for PC1 to be $A 1=3 \mu \mathrm{m}$ and we now calculate the PBG for PC2 with $A 2$ varied in the range $(0.05-0.95) A 1$, while conserving $f$.

For example, for a value of $A 2=(0.5) A 1$ we must reduce the thickness of $D 2_{\mathrm{Si}}$ by a factor of 2 in order to retain the original value of $f$. This procedure allows us to overlap the gap maps for PC1 and PC2 and to define the regions where PBG extension can be expected, as was shown in an earlier section. In Fig. 3(a) the result of merging the gap maps for the PCs with the lattice constants $A 1=3$ and $A 2=(0.83) A 1$ $=2.5 \mu \mathrm{m}$ is shown. Note that all other values used for the analysis were the same as in the previous case, i.e., $n_{\mathrm{Si}}$ $=3.42, n_{\text {air }}=1, m=10$ and $R_{\mathrm{PBG}}=0.999$. The PBGs for PC2 $(A 2=2.5 \mu \mathrm{m})$ are slightly shifted to larger wave numbers in comparison to the PBGs of PC1. As can be seen from Fig. 3(a), five secondary PBGs overlap to form one extended $\mathrm{PBG}$ in the region of $f=0.5-0.62$. Another EPBG is seen in the region of $f=0.05-0.08$.

Once we know the most probable regions for the formation of an EPBG we can construct the gap map for the composite photonic crystal. As in a previous case, we design a CPC which is the combination of PC1 with $A 1=3 \mu \mathrm{m}$ and PC2 with $A 2=2.5 \mu \mathrm{m}$. When performing the gap map calculations we take into account the equality of $f$ for both PCs as was mentioned previously. Next, the reflection spectrum $R$ of the composite $\mathrm{PC}$ is calculated by the transfer matrix method for different $f$ values. To finish, we select only those values of $\widetilde{\nu}$ for which $R>0.999$ allowing us to construct the gap map of the composite PC shown in Fig. 3(b). 

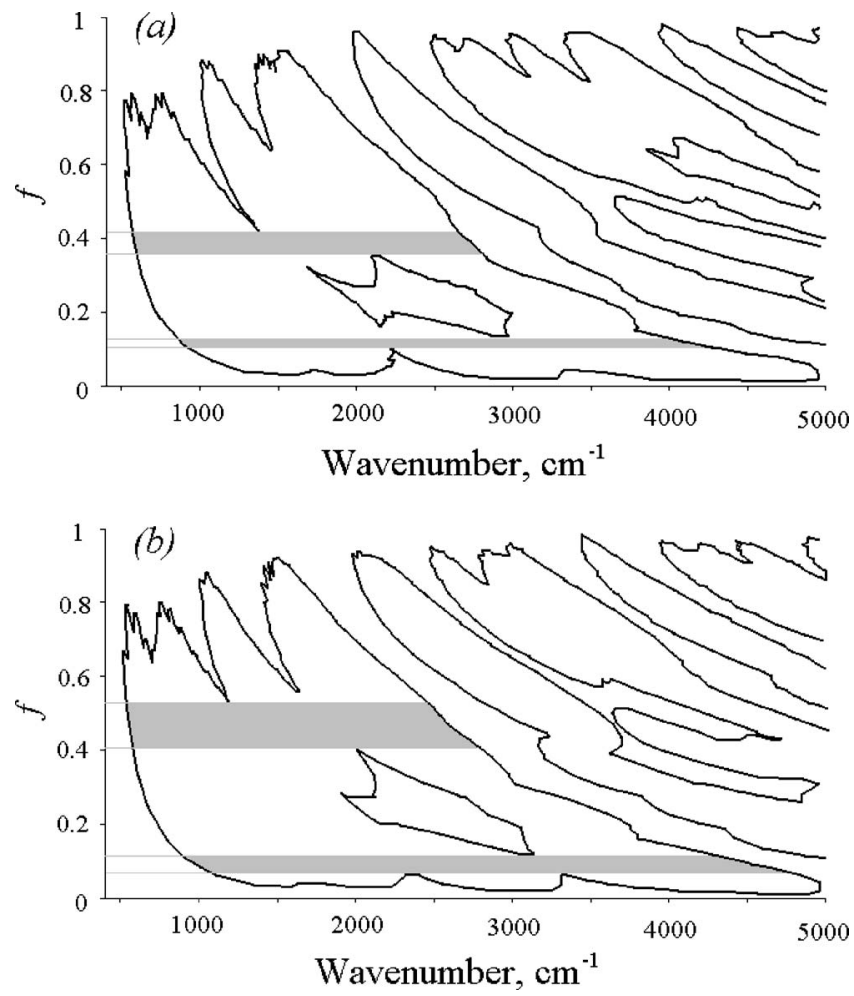

FIG. 4. PBG gap map for composite PC with lattice constants (a) $A 1=3$ and $A 2=2.22 \mu \mathrm{m} \quad[A 2=(0.74(A 1]$ and (b) $A 1=3$ and $A 2=2.13 \mu \mathrm{m} \quad[A 2$ $=(0.71) A 1]$.

From Fig. 3(b), the closed regions representing the PBGs are visible as in the case of a conventional PC. As expected from Fig 3(a), the EPBG regions are located in the areas predicted, in the regions of $f=0.06$ and $f=0.6$. We can also see from this figure that the secondary PBGs are also extended. The main PBG regions also form an extended PBG [Fig. 3(b)] for the composite PC due to the partial overlapping of the main PBGs from Fig. 3(a). However, it can be also seen from Fig. 3(b) that the range of transmission between the main and closest secondary band gap for the CPC is not closed when using PC2 with $A=2.5 \mu \mathrm{m}$. Obviously, the main PBG of PC2 must be shifted to the larger wavenumber range. This can be achieved by a reduction of the value $A$. Reducing the value $A$ further down to $(0.74) A 1$ $=2.22 \mu \mathrm{m}$ leads to the merging of the main PBGs in the composite PC, forming two EPBGs in the regions of $f=0.1$ and $f=0.4$, shaded in Fig. 4(a). In contrast to conventional PC gap maps, regions of transparency may exist in the middle of the extended PBGs in composite PCs. We note that for $f=0.37-0.41$ the region of the predicted EPBG will exist over a fairly narrow interval of $f$ values. The $f$ value, as is well known, is a parameter of the geometrical sizes of the periodic PC structure. Therefore, even small deviations from the required values of these parameters may result in fluctuations in $f$, which, if large enough, could prevent the formation of the EPBG altogether, as seen from Fig. 4(a).

Clearly, the range of $f$ values over which an EPBG can exist must be as wide as possible for a CPC, in order to allow it to be fabricated easily. Bearing this assertion in mind, we can determine the optimal design parameters for the fabrication of the CPC by reducing $A$ further to $A 2=(0.71) A 1$
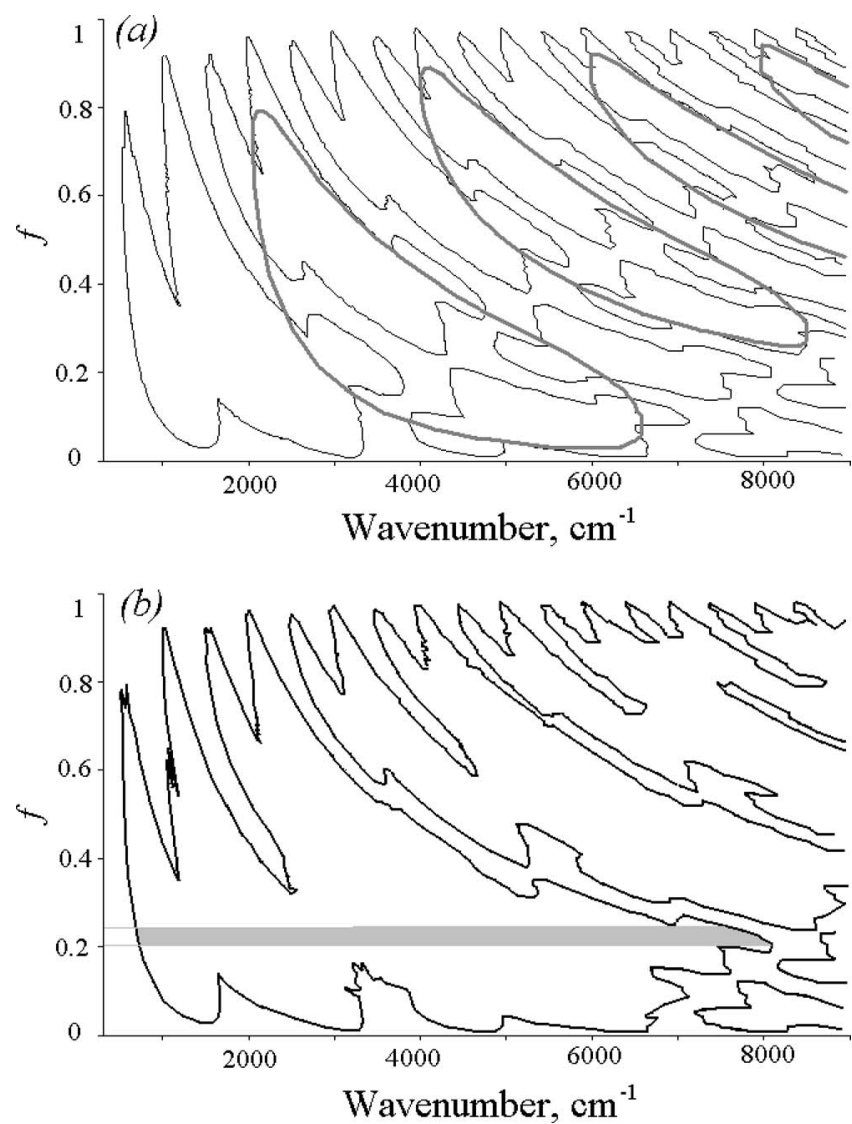

FIG. 5. PBG gap map for composite PC with lattice constants (a) $A 1=3$ and $A 2=1.5 \mu \mathrm{m}[A 2=(0.5) A 1]$, the thick line corresponds to the main and several secondary band gaps for a PC with $A=0.72 \mu \mathrm{m}[A 3=(0.24) A 1]$. (b) $A=3,1.5$, and $0.72 \mu \mathrm{m}, m_{1,2,3}=10$.

$=2.13 \mu \mathrm{m}$, Fig. 4(b). From Fig. 4(b), the range of $f$ values almost doubles. Moreover, another EPBG is also seen in Figs. 4(a) and 4(b) for values of $f=0.08-0.11$, although the range of $f$ for this EPBG is small. Further decreases in the value of $A 2$ to (0.5)A1 leads to the merging of one secondary band gap and two main ones, see Fig. 5(a), for $f=0.22$, despite the fact that there is a wide transparency regions between these PBGs [Fig. 1(a)]. In order to achieve the maximum PBG extension the aim must be to join the main PBGs at their widest region, which is the range of $\lambda / 4$ layers $(f$ $=0.226$ ) for this type of PC.

As a final example of the use of the technique, it can be shown that a composite PC can be further combined with another conventional PC. As can be seen in Fig. 5(a), for $f$ $=0.22$ in the range of $\widetilde{\nu}=3800-5000 \mathrm{~cm}^{-1}$ there is a region of transparency. By analogy with the previous case, we can locate the corresponding PC with a main PBG which will cover this transparent region. From Fig. 5(a) we can see that by using the gap map of PC3 with $A=0.72 \mu \mathrm{m}$ (thick line) we may be able to close the regions of transparency for the $f$ values required. After calculating the gap map for the modified composite PC, we obtain an EPBG which is a combination of the main PBG of PC3 and the secondary PBGs of the original composite PC as shown in Fig. 5(b). The EPBG of the composite PC is in the range $\widetilde{\nu}=800-7800 \mathrm{~cm}^{-1}(\lambda$ $=1.2-12.5 \mu \mathrm{m})$. It is worth noting that changing the order of 
the conventional PC and composite PC in the merging and mapping process has no effect on the gap map of the final three elements composite PC.

Returning to the earlier published results on PBG extension, ${ }^{7-9}$ we can comment that introducing a thickness fluctuation, which follows some particular distribution, to one or both layers in a binary layered structure is equivalent to introducing a change in the $A$ or $f$ parameters of the PC. In this study, we are not going to estimate the advantages and disadvantages of one or other approaches for the design of PCs with an extended PBG. However we would like to note that the approach suggested here cannot be related to disorder induced extension, on the contrary, we introduce ordering into the construction of the PC in order to localize light and to engineer its optical characteristics. The analysis of the influence of the number of periods as well as the optical contrast on the formation of EPBGs will be consider in a future paper.

\section{CONCLUSION}

A method for the extension of photonic band gaps by designing a composite periodic structure consisting of conventional PCs with different lattice constants and filling factors is presented. The design of the PC structure is done by merging the gap maps for two (or more) PCs and by enclosing the transparent regions of the first PC by the PBGs of the second PC. Designing composite PCs with different lattice constants and a constant filling factor gives more flexibility during the design flow than using PCs with different filling factors and a constant lattice constant. The gap maps of a composite $\mathrm{PC}$ for a representative $\mathrm{Si}$-air system are calculated from the near-infrared to the far-infrared range in order to demonstrate the method.

\section{ACKNOWLEDGMENTS}

This work has been supported by the Science Foundation Ireland Basic Research Program (Grant No. 04/BR/P0698) and Russian Program Scientific School - 758.2003.2. The authors would like to thank Ekaterina Astrova for useful discussion and Seamus McGettrick, Siobhan O'Neill, and Marco Cimino for gap map calculations.

${ }^{1}$ E. Yablonovitch, Phys. Rev. Lett. 58, 2059 (1987).

${ }^{2}$ J. D. Joannopoulos, R. D. Meade, and J. N. Winn, Photonic Crystals: Molding the Flow of Light (Princeton University Press, Singapore, 1995).

${ }^{3}$ M. Born and E. Wolf, Principles of Optics, 6th edition, (Pergamon, New York, 1980).

${ }^{4}$ R. M. A. Azzam and N. M. Bashara, Ellipsometry and Polarized Light (North-Holland, Amsterdam, The Netherlands, 1977).

${ }^{5}$ V. A. Tolmachev, T. S. Perova, and K. Berwick, Appl. Opt. 42, 5679 (2003).

${ }^{6}$ D. N. Chigrin, A. V. Lavrinenko, D. A. Yarotsky, and S. V. Gaponenko, J. Lightwave Technol. 17, 2018 (1999).

${ }^{7}$ D. Zhang, W. Hu, Y. Zhang, Z. Li, B. Cheng, and G. Yang, Phys. Rev. B 50, 9810 (1994).

${ }^{8}$ D. Zhang, Z. Li, W. Hu, and B. Cheng, Appl. Phys. Lett. 67, 2431 (1995).

${ }^{9} \mathrm{H}$. Li, H. Cheng, and X. Qiu, Physica B 279, 164 (2000).

${ }^{10} \mathrm{P}$. Sheng, B. White, Z. Q. Zhang, and G. Papanicolaou, Scattering and Localization of Classical Waves in Random Media, edited by P. Sheng (World Scientific, Singapore, 1990), p. 563.

${ }^{11}$ A. Kondilis and P. Tzanetakis, Phys. Rev. B 46, 15426 (1992); J. Opt. Soc. Am. A 11, 1661 (1994).

${ }^{12}$ A. R. McGurn, K. T. Christensen, F. M. Mueller, and A. A. Maradudin, Phys. Rev. B 47, 13120 (1993).

${ }^{13}$ V. A. Tolmachev, T. S. Perova, J. Pilyugina, and R. A. Moore, Opt. Commun. (to be published).

${ }^{14}$ W. H. Southwell, Appl. Opt. 38, 5464 (1999). 